\title{
Micro-CT Imaging for Understanding the Formation of Impact Spherules and Their Compositional Heterogeneity: A Case Study from Lonar Impact Crater, India
}

\author{
Rahul Das Gupta ${ }^{1}$, Ramananda Chakrabarti ${ }^{1}$ \\ 1. Centre for Earth Sciences, Indian Institute of Science, Bangalore, India
}

Impact craters are ubiquitous features on planetary surfaces in the Solar System and indicate that planetary collisions were a dominant process in the early Solar System, leading to the accretion and growth of planets. Impact cratering excavates sub-surface material and these depressions on planetary surfaces have also been the preferred landing site on planetary bodies, e.g., the Curiosity and Spirit rovers landed on the Gale and Gusev craters, respectively, on Mars. Among nearly 190 discovered craters on Earth [1], the Lonar Crater in India presents a unique opportunity to understand cratering processes on other planetary bodies because the target rock is basalt [2] which is the dominant lithology on the surface of most inner Solar System planetary bodies. The clues to understanding the geochemical effects of cratering lie in the composition of impactites, which are formed by cooling and solidification from the melt or the vapor, generated due to the high energy impact [3]. Among the impactites, impact spherules, having aerodynamic shapes with longest diameter $<1 \mathrm{~mm}$ [4], preserve the geochemical and isotopic signatures of the impactor, which is usually vaporized upon impact. At Lonar Crater, some spherules (Group 1a) preserve chemical traces of the impactor at Lonar in the form of higher concentrations of Platinum Group Elements (Fig. 1a) compared to the target rock; the Group 1b spherules show signatures which are explained by the melting of the basement beneath the target Deccan basalts [5]. In addition, some spherules show concentrations of volatile elements that are higher than any of the end-members involved in the impact cratering process at Lonar, indicating condensation from a volatile element-enriched part of the melt/vapor plume [5].

Such chemical heterogeneity in the spherules indicates that they are not formed by a uniform physical mechanism. There is clearly a density difference among the spherules at Lonar Crater, based on their variable elemental compositions. In this study, the internal 3-dimensional morphology of the spherules was imaged using X-ray Micro - computed tomography (Micro-CT) to characterize the density differences within the Lonar spherules. This combined approach involving Micro-CT imaging and geochemical analyses of the same spherules will provide a better understanding of the mechanism of chemical fractionation within spherules.

Spherules were hand-picked from surface sediments collected from near the Lonar Crater. Micro computed tomography of a set of 3 such samples was carried out using an X-ray microscope (Versa XRM 500 , Xradia) with isotropic voxel size of $3.5 \mu \mathrm{m}$. The resultant image was acquired using 1000 projections, where the exposure time for each projection was 1 second. The source voltage used was $90 \mathrm{kV}$ and the power was $8 \mathrm{~W}$. Representative 2-d images (e.g., Figure 1c), with one voxel thickness, were taken at XY, $\mathrm{YZ}$ and $\mathrm{XZ}$ sections.

The preliminary Micro-CT scans of the spherules show that there is significant compositional and morphological heterogeneity within the samples. There is varying abundance of void spaces within them, indicated by the dark spots (spherule on the left, in Figure 1b). These possibly signify incorporation of varying proportions of the vapor plume, during condensation from the melt. This, in turn, can have implications for concentrations of volatile elements. In addition to the low density dark spots, there are 
high density bright spots as well, which are mostly at the rims of the samples (Figure 1b, 1c). The occurrence of the bright spots is significant, because it gives an insight to the mechanism of capturing the meteoritic signatures, typically characterized by higher concentrations of transition metals, within the spherules. A study of the chemical composition of the same samples would be useful to correlate with the actual composition of the dark and bright patches and hence understand the possible mechanisms of entrapment of volatile elements or meteoritic signatures. A similar approach can be suggested for samples to be returned in the future from planetary surfaces because such samples are likely to enriched in impactites.

\section{References:}

[1] S. Hergarten and T. Kenkman, Earth and Planetary Science Letters 425 (2015), p. 187.

[2] K. Fredrikkson et al, Science 180 (1973), p. 862.

[3] B.C. Johnson and H.J. Melosh, Icarus 217 (2012), p. 416.

[4] B.P. Glass and B.M. Simonson, Elements 8 (2012), p. 43.
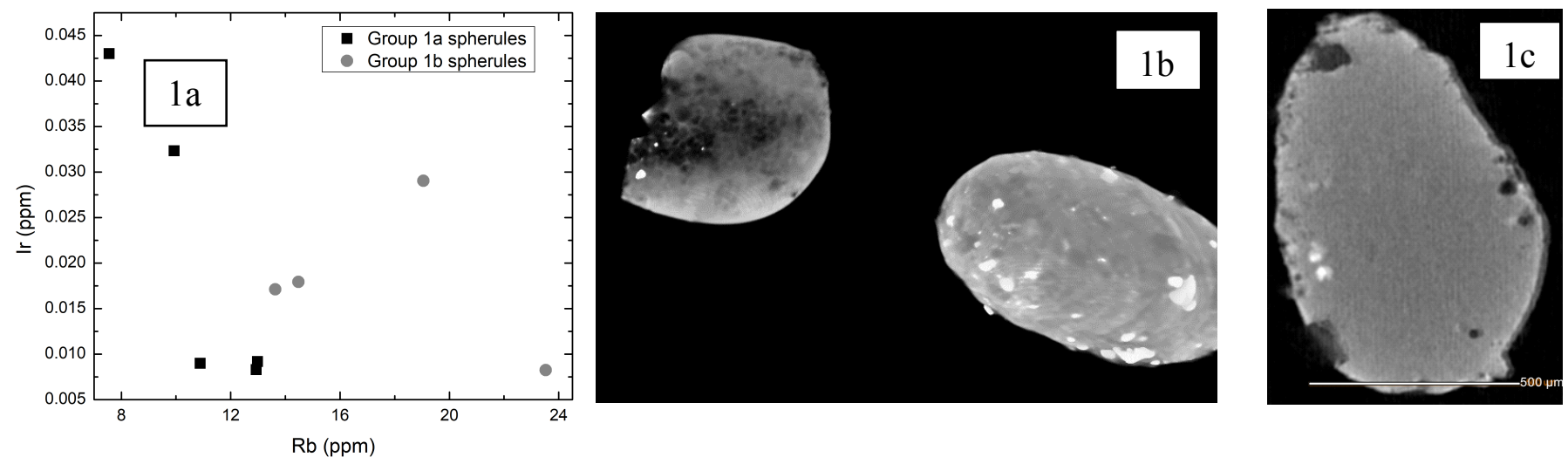

Figure 1a. Chemical heterogeneity among impact spherules at Lonar - Group 1a has higher concentrations of Iridium (PGE), whereas Group 1b has higher concentrations of Rb (LILE) (Data from [5]). Figure 1b. 3-d Micro - CT image of spherules - hollow nature of a spherule, with lot of void spaces (dark patches) on the left, as compared to the one on right. Figure 1c. Chemical heterogeneity manifested in XZ section of a Micro-CT image of a spherule, in the form of dark and bright patches. 